El rol de los actores tecnológicos en la privatización de la universidad

en contexto de pandemia: respuestas inmediatas para grandes transformaciones

Lucía Trotta, Daniela Atairo

Trayectorias Universitarias, 7 (12), e048, 2021

ISSN 2469-0090 | https://doi.org/10.24215/24690090e048

https://revistas.unlp.edu.ar/TrayectoriasUniversitarias

Universidad Nacional de La Plata

La Plata | Buenos Aires | Argentina

\title{
El rol de los actores tecnológicos en la privatización de la universidad en contexto de pandemia: respuestas inmediatas para grandes transformaciones
}

\author{
The role of technological actors in the privatization of higher education \\ in a pandemic context: immediate responses to major transformations
}

\section{Lucía Trotta}

https://orcid.org/0000-0001-8373-2730

luciatrotta.2@gmail.com

Facultad de Humanidades y Ciencias de la Educación Universidad Nacional de la Plata

Instituto de Estudios y Capacitación

- Conadu | Argentina

\author{
Daniela Atairo \\ https://orcid.org/0000-0002-2887-3407 \\ atairodaniela@gmail.com \\ Facultad de Humanidades y Ciencias de la \\ Educación Universidad Nacional de la Plata | \\ Instituto de Estudios y Capacitación \\ - Conadu | Argentina
}

\section{RESUMEN}

Este artículo contribuye al debate sobre los efectos de la pandemia en la educación superior. Se analizan las diferentes alianzas público-privadas que involucran a agentes tecnológicos comerciales para dar respuesta a la continuidad de la enseñanza en las universidades de la región de América Latina y el Caribe. Además, se analiza el papel que tienen organismos internacionales y los agentes tecnológicos globales en la construcción del discurso de la "gran oportunidad de innovación" que pone en cuestión el modelo de universidad presencial dominante en América Latina. Desde una perspectiva teórica que contempla la complejidad del fenómeno de la privatización educativa y que reconoce que en estos procesos impulsados por diversos actores globales, tienen un rol central los estados nacionales y los diferentes gobiernos a la hora de materializar estas tendencias. El estudio se basó en un análisis documental que supuso relevar discursos y acciones de diferentes actores que asumieron centralidad en el contexto de pandemia en el nivel universitario.

\section{ABSTRACT}

This article contributes to the debate on the effects of the pandemic on higher education. It analyses the different public-private alliances that involve technological commercial agents to respond to the continuity of teaching in universities from Latin American and Caribbean region. It also analyses the role that international organisms and technological agents have in the construction of "big opportunity of innovation" discourse that challenges the in-person university model dominant in Latin America. It starts from a theoretical perspective that contemplates the complexity of educational privatization phenomenon and recognizes that, in these processes driven by various global actors, national states and different governments play a central role when it comes to materialize this trends. The study was based on a documentary analysis that involved gathering discourses and actions from different actors that assumed centrality in the context of the pandemic at the university level.

PALABRAS CLAVE

Privatización, Mercantilización Educación Superior, Pandemia.

\section{KEY WORDS}

Privatization Commodification, Higher Education, Pandemic. 


\section{INTRODUCCIÓN}

Este trabajo presenta los resultados de la investigación "Procesos de privatización y mercantilización de las universidades de América Latina y el Caribe en contexto de pandemia" que, a su vez, se inscribe en una línea de investigación sobre los procesos de privatización y mercantilización de la educación superior y el conocimiento en América Latina' . En particular, en este escrito focalizamos en uno de los aspectos emergentes que vino a consolidar y profundizar dimensiones ya presentes en la región relativas al posicionamiento de actores tecnológicos comerciales en el campo de la política universitaria.

La pandemia nos enfrentó a un tiempo inédito e impensado donde gran parte de las actividades humanas se vieron trastocadas, dando paso a nuevos modos de encontrarse, relacionarse y, también, de investigar, enseñar y aprender. El estudio se centra en analizar los procesos que se desplegaron en el afán por sostener la docencia universitaria con universidades cerradas, en especial en las dimensiones que tensionan la efectivización de la Educación Superior (ES) como un bien público social, un derecho humano y universal y un deber del Estado, tal como fuera declarado en la Conferencia Regional de Educación Superior (CRES) de Cartagena en el año 2008 y ratificado en Córdoba en el 2018.

Desde una mirada que contempla la complejidad, transversalidad y multipolaridad del fenómeno, entendemos la privatización, en términos generales, como la mayor participación de agentes privados en la titularidad, provisión, gestión y financiamiento del sistema educativo. Mientras que la mercantilización supone la introducción de normas, valores y principios propios del mercado, como el de competencia y elección, en el sector público. Ambos procesos se presentan como dos

El informe se basa en un análisis sistemático del informe de "Coyuntura Latinoamericana" elaborado por el Observatorio Latinoamericano de Políticas Educativas realizado por la oficina regional de la IEAL; en la indagación del sitio web de SITEAL (UNESCO) que puso a disposición noticias sobre iniciativas gubernamentales difundidas en los sitios de internet y cuentas de redes sociales de organismos oficiales de los países; en búsquedas en internet sobre documentos y actividades de varias organizaciones pertinentes por el tema en cuestión y en notas de prensa sobre núcleos significativos identificados, además de un conjunto de entrevistas vía formulario a dirigentes sindicales de nivel universitario que aportaron información y opiniones. El análisis se realizó durante mayo-diciembre, meses en los cuales la orientación de las medidas fue cambiando en paralelo a la extensión y complejización del escenario socio-sanitario. 
caras de la transformación de la educación superior en una mercancía y en estrecho vínculo con los procesos de gobernanza corporativa, donde representantes de corporaciones tienen una participación directa en la definición de la agenda de la educación pública (CLADE, 2014). Esta mirada supone reconocer que estos procesos son impulsados por actores que buscan incidir en la orientación de la política educativa y favorecer las condiciones para la emergencia y consolidación de proveedores privados, desde organismos supranacionales, agentes económicos transnacionales, pero también cabe resaltar el rol de los estados nacionales y los diferentes gobiernos a la hora de materializar estas tendencias (Verger, 2013; Brunner, 2008). La perspectiva de análisis reconoce además que los sistemas de educación superior de la región guardan una gran heterogeneidad, con configuraciones institucionales de diversa trayectoria, principios y criterios organizacionales, por lo que las características intrínsecas respecto de ciertas condiciones sociopolíticas, institucionales, demográficas e históricas de cada caso en cuestión, son un elemento central en la comprensión de los procesos (Moschetti y Gottau, 2015; Verger et. al., 2017).

Es así que este enfoque nos permitió, en primer lugar, identificar que el tipo de respuesta que se configuró en cada sistema de educación superior (SES) de la región con la llegada del COVID-19 fue muy variable y dependió, no sólo de la situación económica, sanitaria y política de cada país, sino también de tres dimensiones: el grado de privatización y mercantilización de la ES, el desarrollo previo de la Educación a Distancia (EaD) y el avance en la ampliación del acceso a la ES y las desigualdades persistentes, que limitan el ejercicio del derecho a la educación en términos de acceso a la universidad y a las tecnologías. Y, en segundo lugar, observar que las acciones implementadas en el marco de una educación remota de emergencia provocada por la pandemia por el cierre de las instalaciones edilicias de las instituciones, han consolidado y actualizado las tendencias de privatización y mercantilización de la ES presentes en la región.

Es así que este enfoque nos permitió, en primer lugar, identificar que el tipo de respuesta que se configuró en cada sistema de educación superior (SES) de la región con la llegada del COVID-19 fue muy variable y dependió, no sólo de la situación económica, sanitaria y política de cada país, sino también de tres dimensiones: el grado de privatización y mercantilización de la ES, el desarrollo previo de la Educación a Distancia (EaD) y el avance en la ampliación del acceso a la ES y las desigualdades persistentes, que limitan el ejercicio del derecho a la educación en términos de acceso a la universidad y a las tecnologías. 
A continuación, presentaremos las principales reflexiones sobre una de las tendencias encontradas: las nuevas alianzas público-privado que emergen durante la pandemia, el rol de los agentes tecnológicos globales en este proceso, y la injerencia en el diseño de políticas universitarias.

\section{Alianzas público-privado: el rol de los agentes tecnológicos en la provisión de la infraestructura necesaria para dar continui- dad educativa en la región.}

Si bien en muchas universidades de la región ya se había iniciado un proceso de adopción de nuevas tecnologías, la forma repentina y acelerada que exigió el contexto de pandemia, requirió distintos tipos de alianzas con actores comerciales tecnológicos con el fin de garantizar rápidamente infraestructura, conectividad, dispositivos, entornos y recursos virtuales que permitieran pasar a una enseñanza remota de emergencia. Así, las principales iniciativas estuvieron orientadas a favorecer la instalación de soluciones tecnológicas de emergencia para dar continuidad inmediata a la enseñanza en un contexto de universidad sin universidades.

En este marco, los organismos internacionales promovieron la participación de diversos actores empresariales en América Latina (Bonilla, 2020). Es así que el Banco Interamericano de Desarrollo (BID) sugería hacia mayo del 2020 "buenas prácticas para la continuidad educativa" como establecer alianzas con las empresas editoriales y de telecomunicaciones y con proveedores de internet. O la UNESCO, quien propició la Coalición Mundial para la Educación, una asociación multisectorial para asegurar una adecuada educación a distancia a todo el alumnado donde participan socios como organizaciones multilaterales internacionales, organizaciones de la sociedad civil, organizaciones sin ánimo de lucro, empresas del sector privado y otro tipo de organizaciones y redes. Por su parte, desde IESALC -organismo específico de la UNESCO para la ES-, se promovió la mejora de las condiciones para acceder a equipamientos $y$, sobre todo, a paquetes de conectividad móvil que facilitaran que Ixs estudiantes y docentes mejoren su capacidad tecnológica, así como propusieron a los gobiernos promover que las compañías de telecomunicaciones ofrezcan gratuidad en el uso de datos para aplicaciones y servicios educativos (mayo 2020).

Las características que asumen las acciones orientadas a generar mejores condiciones para la enseñanza remota en un contexto de emergencia socio-sanitaria han colocado a las tecnologías en el centro de la cuestión, profundizando así procesos de privatización, en tanto el sector privado cumple un papel central en la provisión de los servicios esenciales que se constituyen en una condición para acceder al derecho a la educación. 
Las características que asumen las acciones orientadas a generar mejores condiciones para la enseñanza remota en un contexto de emergencia socio-sanitaria han colocado a las tecnologías en el centro de la cuestión, profundizando así procesos de privatización, en tanto el sector privado cumple un papel central en la provisión de los servicios esenciales que se constituyen en una condición para acceder al derecho a la educación.

Del relevamiento realizado, se observa como tendencia general, que las acciones se centraron en, por un lado, ampliar y mejorar la conectividad y los dispositivos de estudiantes y docentes, y por otro, poner a disposición de las instituciones de educación superior (IES) que carecían de plataformas de educación virtual propias, mecanismos y recursos tecnológicos para la impartición de cursos a distancia. Aunque las situaciones son muy heterogéneas al interior de cada país y entre los países de la región. En este punto, fue medular la situación previa respecto del desarrollo de la EaD en cada país y sus IES, como también el formato que había asumido hasta este momento: más volcado a la compra de servicios a empresas internacionales, más orientado al desarrollo de software o tecnologías propias o al uso de software libre, como en el caso de argentina.

Williamson y Hogan (2020) han observado a nivel mundial la presencia desde proveedores de productos específicos hasta redes o coaliciones intersectoriales (que implican la mediación de estamentos gubernamentales, agencias multilaterales, empresas privadas y diferentes tipos de organizaciones intermedias), que se convirtieron en actores esenciales al resolver la paralización de la educación en un proceso que condensó aspectos previos, pero también habilitó la configuración de una escena a futuro.

En el caso regional, se ha registrado que las coaliciones gubernamentales e institucionales con actores comerciales tuvieron diferentes propósitos y diferente grado de complejidad en función de la cantidad de los actores que participaron. Los agentes tecnológicos comerciales establecieron alianzas con gobiernos e instituciones para garantizar conectividad y dispositivos, a partir de variados formatos. Se destacan los subsidios directos a las instituciones (Perú), entrega de dispositivos tecnológicos (Ecuador, Paraguay), convenios con compañías de telecomunicaciones para planes de conectividad y acceso a dispositivos con precios preferenciales (Ecuador, República Dominicana, Colombia), acuerdos con empresas de telecomunicaciones para liberar el consumo de datos en los sitios web educativos (Bolivia, Argentina, Panamá). Otras alianzas tenían como propósito garantizar Entornos Virtuales de Enseñanza y Aprendizaje y otros recursos digitales. En este punto las alianzas y respuestas que se fueron construyendo tuvieron un fuerte componen- 
te institucional, lo que potenció la heterogeneidad y complejidad del tipo de respuestas hacia el interior de los sistemas. A nivel de las instituciones, en gran parte de los países de la región como El Salvador, Perú, República Dominicana, Colombia, se reiteran experiencias de instituciones, que ya contaban con cierto grado de desarrollo y experiencia en plataformas educativas, pero que, ante la eventualidad, reforzaron sus alianzas y compra de servicios a empresas como Microsoft, Google, Blackboard e llumnoii , y con otras plataformas especializadas. A nivel gubernamental, se presentan casos de alianzas más integrales como el caso de Chile, donde la Subsecretaría de Educación Superior realizó un convenio con Google, para poner a disposición de las instituciones de Educación Superior que no contaran con plataformas y/o herramientas digitales, la plataforma Google Suite.

También se observan coaliciones más complejas que nuclean a organismos internacionales, empresas tecnológicas, ministerios y universidades públicas en vistas de brindar capacitación a docentes universitarios sobre enseñanza virtual. Por ejemplo, el IESALC- UNESCO fue seleccionado en convocatoria abierta del Ministerio de Educación del Perú y el Banco Interamericano de Desarrollo para implementar el Programa de apoyo al diseño e implementación de estrategias para la continuidad pedagógica, impulsado en el marco del PMESUT (Programa para la Mejora de la Calidad y Pertinencia de los Servicios de Educación Superior Universitaria y Tecnológica a Nivel Nacional) y de la DIGESU del Ministerio de Educación de Perú . El IESALC-UNESCO, que viene desarrollando proyectos con Microsoft en la región, es el actor que promueve y garantiza los vínculos con el Ministerio de Educación de Perúiii y un conjunto de universidades públicas peruanas. Esta coalición compleja entre organismos internacionales, una empresa tecnológica como Microsoft, un ministerio de educación y un conjunto de universidades, se plasmó en acciones concretas en Perú como "piloto" para ser implementado posteriormente en otros países de la región.

\section{También se observan coaliciones más complejas que nuclean a organismos internacionales, empresas tecnológicas, ministe- rios y universidades públicas en vistas de brindar capacitación a docentes universitarios sobre enseñanza virtual.}

ii llumno es una empresa que provee servicios y soluciones a IES en el segmento de educación virtual en relación a entornos educativos virtuales, gestión de la matrícula, marketing, etc.

iii IESALC UNESCO. (12 de agosto de 2020). UNESCO IESALC proporcionará asistencia técnica para fortalecer la educación superior en el Perú. Recuperado de: https://www. iesalc.unesco.org/2020/08/12/unesco-iesalc-proporcionara-asistencia-tecnica-para-fortalecer-la-educacion-superior-virtual-en-el-peru/ 
Y, finalmente, otra forma de alianza que se observa se asienta en la expansión de los denominados MOOC (cursos en línea, masivos y abiertos), una modalidad de formación virtual acotada orientada a la empleabilidad y de certificación en línea. Si bien, es una propuesta con un alto grado de expansión en tiempos pre-pandémicos, asumen en este contexto una visibilidad y desarrollo más marcado en la región de la mano de alianzas gubernamentales. Un ejemplo de ello son las iniciativas de la plataforma COURSERA que, dentro de su línea para gobiernos, llevó adelante alianzas con los gobiernos de Colombia, Costa Rica, Guatemala, Panamá y la Ciudad Autónoma de Buenos Aires en Argentina. El programa supone la oferta de cursos gratuitos con certificación a una cantidad limitada de usuarios, en algunos casos estudiantes de IES en otros, población en general, con la idea de capacitar en habilidades requeridas por el mercado laboral y brindar herramientas de profesionalización, en un contexto de fuerte pérdida del empleo.

Es decir, en un contexto de reducción de matrícula de las instituciones universitarias, se expanden la oferta de formación que se presenta, aún sin explicitarlo, como contrapuesto al modelo de universidades tradicionales latinoamericanas.

Es decir, en un contexto de reducción de matrícula de las instituciones universitarias, se expanden la oferta de formación que se presenta, aún sin explicitarlo, como contrapuesto al modelo de universidades tradicionales latinoamericanas.

\section{Alianzas público-privado: el rol del sector privado en la orientación del cambio universitario}

En América Latina, como señalamos, se conformaron coaliciones con diferentes grados de complejidad para fomentar el uso de las tecnologías de la educación como respuesta de emergencia en el nivel universitario. Las empresas tecnológicas operan a muy distintas escalas: pueden ir desde la participación en complejas coaliciones internacionales de alto nivel hasta coaliciones entre empresas y gobierno, o entre empresas e instituciones en el marco de la autonomía de las universidades. De allí que, el protagonismo que cobraron en este escenario diferentes actores comerciales los posicionó como interlocutores en la orientación del cambio universitario que se está promoviendo para los sistemas de educación superior. De este modo, en la medida en que el sector privado ofrece soluciones rápidas a problemas públicos se acelera el proceso de privatización de las políticas públicas ya presente en la región.

Las soluciones que se presentan al gran problema del parate edu- 
cativo tienen para estas empresas un beneficio comercial en lo inmediato, con una ampliación del mercado, pero también una proyección de extender sus ganancias al ampliar el mercado para sus productos, plataformas y servicios en el marco de una gran transformación en la educación universitaria basada en la EaD multimodal. En este sentido, la educación a distancia se percibe no sólo como un formato para impartir educación, sino que también se consolida como un segmento de mercado para la realización de transacciones de los grandes actores comerciales transnacionales.

\begin{abstract}
Las empresas tecnológicas operan a muy distintas escalas: pueden ir desde la participación en complejas coaliciones internacionales de alto nivel hasta coaliciones entre empresas y gobierno, o entre empresas e instituciones en el marco de la autonomía de las universidades. De allí que, el protagonismo que cobraron en este escenario diferentes actores comerciales los posicionó como interlocutores en la orientación del cambio universitario que se está promoviendo para los sistemas de educación superior.
\end{abstract}

Cabe señalar, que la UNESCO-IESALC ha ocupado un lugar central como organización internacional en el asesoramiento en la región en lo referente a la virtualización de la enseñanza en la educación superior frente a la emergencia del COVID-19. Es uno de los actores principales que promueve un nuevo modelo de organización híbrido de los procesos de enseñanza y aprendizaje. Incluso, promoviendo discusiones sobre la inteligencia artificial como parte de la agenda de la educación superior pública en América Latina a partir de un proyecto en común con Microsoft que impulsa la transformación educativa y que tiene como referentes a varias universidades privadas y algunas públicas en la región, junto con la presencia de grandes actores económicos como Kroton Educational y el Grupo Laureate (Braverman, 2020).

Es decir, una gran transformación del nivel está siendo promovida por organismos internacionales y actores comerciales globales en base a la idea de convertir esta crisis en una oportunidad para la innovación, que implica, por ejemplo, repensar los diseños tradicionales de la educación superior e instalar modelos híbridos o 100\% virtuales. Este escenario modifica sustancialmente el desarrollo de la EaD en la región porque las propuestas que predominaban eran las unimodales, de carácter semivirtual con la obligatoriedad de instancias formativas presenciales. Muy recientemente se habían comenzado a desarrollar propuestas de multimodalidad habilitadas por nuevas regulaciones, 
especialmente en Colombia, México, Brasil, Costa Rica. Pero en otros países la situación es bien distinta como Bolivia, Perú, Guatemala, Panamá en donde los gobiernos habían frenado el crecimiento de la EaD en el nivel superior (IESALC, 2020; OEl, 2017).

Estos agentes tecnológicos se posicionan así en un lugar estratégico como interlocutores en la definición y orientación de las políticas universitarias, y les permite introducir cambios en los sistemas universitarios de acuerdo con su propia visión y agenda organizativa. Se observa cómo se instala con fuerza una agenda centrada en la formación para la "empleabilidad", la necesidad de flexibilizar formatos y trayectorias, y el desarrollo de habilidades y capacidades acordes al nuevo tecno-capitalismo. Además, se trata de un discurso que consolida la idea de la neutralidad de las tecnologías y de la educación como un problema de orden técnico antes que pedagógico y político, y oculta los riesgos en términos de soberanía digital, amenazando la propiedad intelectual de lo que se produce en las universidades. Estas corporaciones tecnológicas que ofrecen soluciones digitales a gran escala, velocidad y, en la mayoría de los casos, de manera gratuita, han recolectado como contrapartida una cantidad colosal de datos. El aluvión de nuevos usuarios ${ }^{\text {iv }}$ y un uso múltiple, continuo e ininterrumpido que propició la situación de pandemia, no solo les permitió obtener ganancias siderales sino también instalarse como "la" opción tecnológica por excelencia ante la crisis y en el mediano y largo plazo; en detrimento de opciones ancladas en el software libre, con la consecuente pérdida de control sobre la información y los datos.

Se observa cómo se instala con fuerza una agenda centrada en la formación para la "empleabilidad", la necesidad de flexibilizar formatos y trayectorias, y el desarrollo de habilidades y capacidades acordes al nuevo tecno-capitalismo. Además, se trata de un discurso que consolida la idea de la neutralidad de las tecnologías y de la educación como un problema de orden técnico antes que pedagógico y político, y oculta los riesgos en términos de soberanía digital, amenazando la propiedad intelectual de lo que se produce en las universidades.

iv Microsoft y Google son los actores tecnológicos comerciales que más han ampliado su presencia y expansión en la región. En algunos casos, para darle curso a dicha oferta se requiere que la institución adherida y cada docente o estudiante mediante una cuenta institucional acceda de forma gratuita a los productos, evitando los procesos de contratación que suelen implicar las compras de tecnologías de la educación y convirtiéndose así en los principales proveedores de software educativo del mundo, ampliando la base de usuarios en el segmento estudiantil y abriendo nuevas posibilidades de extracción de datos (Williamson y Hogan, 2020). 
La virtualización forzosa se constituyó en el escenario donde el modelo universitario presencial que predominaba en América Latina se puso en cuestión. La velocidad de ese cuestionamiento no deja de sorprender. Del estudio realizado se destaca como tendencia general la profundización de los procesos de privatización de la educación superior en contexto de pandemia que supuso la consolidación de procesos previos, así como la emergencia de nuevas formas. Al mismo tiempo, se reconoce la fuerte heterogeneidad que se presenta entre los países de la región, que se expresaron con diferente amplitud e intensidad en función de las características de las configuraciones institucionales nacionales, más volcadas al mercado o con mayor tradición público- estatal.

En el marco del viraje hacia la educación remota de emergencia se propiciaron alianzas de los gobiernos con proveedores comerciales de servicios tecnológicos, así como la configuración de coaliciones multisectoriales, que otorgaron las bases materiales necesarias para sostener el vínculo pedagógico y el trabajo docente. Ello supuso para estos grandes actores comerciales ganancias económicas que se potenciarán a futuro en un contexto donde ha tomado fuerza el discurso de la "gran oportunidad para la innovación". Estos agentes comerciales que aparecen como los "salvadores" de la educación universitaria se posicionan en un lugar estratégico en la definición y orientación de las políticas públicas e institucionales y en la promoción de modelos universitarios basados en la multimodalidad de la educación a distancia. De allí que se observa una privatización de la política pública en la medida en que el sector empresarial incorpora temas a la agenda de la ES que ponen en cuestión el modelo universitario presencial predominante en América Latina. En este escenario, emerge la instalación de un modelo multimodal de EaD que implica, por un lado, posicionar a las tecnologías en un lugar hegemónico respecto de la problemática del sector y, por otro, dar curso a la preocupación por la empleabilidad y la necesidad de una formación para el tecnocapitalismo.

En este sentido, la privatización no solo está orientada por el mero lucro, hay además una búsqueda constante por generar las condiciones culturales y sociales para construir una sociedad de mercado. De este modo, se proponen modelos de ES que cuestionan aquellos con fuerte arraigo en las sociedades latinoamericanas, despojándolos de todo aspecto político. Queda por ver, en el marco de estas fuertes tensiones, cómo se recrea el sentido público de la universidad en la región, que se oriente hacia instituciones más plurales, diversas y democráticas. 


\section{BIBLIOGRAFÍA}

Bonilla, G. (16 de noviembre de 2020). Monitoreo de soluciones digitales y de educación a distancia [PPT]. Observatorio Latinoamericano de Políticas Educativas. Recuperado de: https://www.observatorioeducacion.org/noticias/america-latina-dialoga-sobre-avance-de-la-privatizacion-y-el-comercio-educativo-en-el

Braverman, L. [UNESCO IESALC]. (27 de octubre de 2020). Lanzamiento de la fase de capacitación docente en el Perú [Video]. Recuperado de: https://www. youtube.com/watch?v=L-qEkCCoHs8\&feature=youtu.be

Brunner, J.J. (2008). El sistema de educación superior en chile: un enfoque de economía política comparada en Avaliação, Campinas; Sorocaba, SP, v. 13, n. 2, p. 451-486, jul. 2008

CLADE (2014). Mapeo sobre tendencias de la privatización de la educación en américa latina y el caribe. Campaña Latinoamericana por el Derecho a la Educación.

Courtney, B. y J. Salmi (18 de abril de 2020). Poniendo la equidad en el corazón de la educación superior.

Gottau, V. y M. Moschetti (2015). El Sistema Educativo argentino. Entre la Privatización interna y la Privatización abierta: trayectorias divergentes y desigualdades. Cadernos de Pesquisa: Pensamento Educacional, (10), 26, 182-202

Internacional de la Educación - América Latina (IEAL) (2021). Tendencias: situación laboral y educativa en América Latina en el contexto de la pandemia Covid-19

OEI (2017). La educación a distancia en la educación superior en América Latina.

Salmi, J., Arnhold, N. and Bassett, R. (junio 2020). El Gran Lobo Malo se mueve hacia el sur: Cómo afecta COVID-19 a la financiación de la educación superior en los países en desarrollo, Publicado en Education for Global Development. Disponible: https://blogs.worldbank.org/es/education/el-gran-lobo-malo-semueve-hacia-el-sur-como-afecta-covid-19-la-financiacion-de-la

UNESCO/IESALC (2020). Hacia el acceso universal a la educación superior: tendencias internacionales.

Verger, A. (2013). Políticas de mercado, Estado y universidad: hacia una conceptualización y explicación del fenómeno de la mercantilización de la Educación Superior. Revista de Educación, 360, pp. 268-291. 
Verger, A.; Moschetti, M.; Fontdevila, C. (2017). La privatización educativa en América Latina: Una cartografía de políticas, tendencias y trayectorias. Universitat Autónoma de Barcelona /IE

Williamson B. and A. Hogan (2020). La comercialización y la privatización en y de la educación en el contexto de la Covid-19. Internacional de la Educación. 